\title{
Some spectral formulas for functions generated by differential and integral operators in Orlicz spaces
}

\author{
Bang H.H. ${ }^{1, \bigotimes}$, Huy V.N. ${ }^{2,3}$
}

In this paper, we investigate the behavior of the sequence of $L^{\Phi}$-norm of functions, which are generated by differential and integral operators through their spectra (the support of the Fourier transform of a function $f$ is called its spectrum and denoted by $\operatorname{sp}(f)$ ). With $Q$ being a polynomial, we introduce the notion of $Q$-primitives, which will return to the notion of primitives if $Q(x)=x$, and study the behavior of the sequence of norm of $Q$-primitives of functions in Orlicz space $L^{\Phi}\left(\mathbb{R}^{n}\right)$. We have the following main result: let $\Phi$ be an arbitrary Young function, $Q(\mathbf{x})$ be a polynomial and $\left(\mathcal{Q}^{m} f\right)_{m=0}^{\infty} \subset L^{\Phi}\left(\mathbb{R}^{n}\right)$ satisfies $\mathcal{Q}^{0} f=f, Q(D) \mathcal{Q}^{m+1} f=\mathcal{Q}^{m} f$ for $m \in \mathbb{Z}_{+}$. Assume that $\operatorname{sp}(f)$ is compact and $\operatorname{sp}\left(\mathcal{Q}^{m} f\right)=\operatorname{sp}(f)$ for all $m \in \mathbb{Z}_{+}$. Then

$$
\lim _{m \rightarrow \infty}\left\|\mathcal{Q}^{m} f\right\|_{\Phi}^{1 / m}=\sup _{\mathbf{x} \in \operatorname{sp}(f)}|1 / Q(\mathbf{x})|
$$

The corresponding results for functions generated by differential operators and integral operators are also given.

Key words and phrases: Orlicz space, inequality in approximation, Fourier transform, generalized function.

\footnotetext{
${ }^{1}$ Institute of Mathematics, Vietnam Academy of Science and Technology, 18 Hoang Quoc Viet str., Hanoi, Vietnam

${ }^{2}$ Hanoi University of Science, Vietnam National University, 334 Nguyen Trai str., Hanoi, Vietnam

${ }^{3}$ Thang Long Institute of Mathematics and Applied Sciences, Thang Long University, Nghiem Xuan Yem str., Hanoi, Vietnam $\square$ Corresponding author

E-mail: hhbang@math.ac.vn (Bang H.H.), nhat_huy85@yahoo.com (Huy V.N.)
}

\section{Introduction}

Let $1 \leq p \leq \infty, \varrho>0, f \in L^{p}(\mathbb{R})$ and $\operatorname{sp}(f) \subset[-\varrho, \varrho]$, where $\operatorname{sp}(f):=\operatorname{supp} \widehat{f}$ and $\widehat{f}=\mathcal{F} f$ is the Fourier transform of $f$. Then it is well-known the following Bernstein inequality (see [11,23]): $\left\|D^{m} f\right\|_{p} \leq \varrho^{m}\|f\|_{p}, m=1,2, \ldots$. Bernstein inequality plays an important role in function theory and has various applications. It was studied and developed by many authors, see, e.g., [16-18, 21, 25-27,33]. The following result is an addition of the Bernstein inequality (see [4]). Let $1 \leq p \leq \infty$ and $D^{m} f \in L^{p}(\mathbb{R}), m=0,1,2, \ldots$, then

$$
\lim _{m \rightarrow \infty}\left\|D^{m} f\right\|_{p}^{1 / m}=\sup \{|\zeta|: \zeta \in \operatorname{sp}(f)\} .
$$

This result has the following extensions (see $[5,7])$.

Let $1 \leq p \leq \infty, f \in L^{p}\left(\mathbb{R}^{n}\right)$ and $\operatorname{sp}(f)$ be compact, then

$$
\lim _{|\alpha| \rightarrow \infty}\left(\left\|D^{\alpha} f\right\|_{p} / \sup _{\zeta \in \operatorname{sp}(f)}\left|\zeta^{\alpha}\right|\right)^{1 /|\alpha|}=1 .
$$

$\mathrm{y} \Delta \mathrm{K} 517.9$

2020 Mathematics Subject Classification: 41A17, 26D10, 42B10, 46E30. 
Further, if $1 \leq p \leq \infty, Q$ is a polynomial, $f \in L^{p}\left(\mathbb{R}^{n}\right)$ and $\operatorname{sp}(f)$ is compact, then

$$
\lim _{m \rightarrow \infty}\left\|Q^{m}(D) f\right\|_{p}^{1 / m}=\sup \{|Q(\mathbf{x})|: \mathbf{x} \in \operatorname{sp}(f)\},
$$

where the differential operator $Q(D)$ is obtained from $Q(\mathbf{x})$ by substituting

$$
\mathbf{x} \rightarrow\left(-i \partial / \partial x_{1},-i \partial / \partial x_{2}, \ldots,-i \partial / \partial x_{n}\right)
$$

where $\mathbf{x}=\left(x_{1}, x_{2}, \ldots, x_{n}\right)$.

The novelty of these results is that the behavior of the sequence of norm of derivatives of a function $f$ is directly investigated through its spectrum $\operatorname{sp}(f)$ but not, as usual, through a given compact $K$ containing $\operatorname{sp}(f)$. These results were studied and developed by many authors (see $[1-3,5-7,12-15])$. It is natural to ask what will happen when we replace derivatives by integrals? For $p=2$, V.K. Tuan proved the following result in [31].

Let $f \in L^{2}(\mathbb{R})$ and $\varrho:=\inf \{|\zeta|: \zeta \in \operatorname{sp}(f)\}>0$. Then there exists $I^{m} f, I^{m} f \in L^{2}(\mathbb{R})$ for all $m$, and

$$
\lim _{m \rightarrow \infty}\left\|I^{m} f\right\|_{2}^{1 / m}=\varrho^{-1}, \quad \text { If }(x)=\int_{x}^{\infty} f(y) d y,
$$

the improper indefinite Riemann integral, and $I^{n}=(I)^{n}$. This result of V.K. Tuan was extended in [8] to the case $f \in L^{p}(\mathbb{R}), 1 \leq p \leq \infty$, to the $n$-dimension case and Orlicz spaces in $[9,10]$.

The purpose of this paper is to extend above results to more general cases. With $Q$ being a polynomial, we introduce the notion of $Q$-primitives, which will return to the notion of primitives used in [8] if $Q(x)=x$, and study the behavior of the sequence of norm of $Q$-primitives of functions in $L^{\Phi}\left(\mathbb{R}^{n}\right)$. Moreover, we also investigate the behavior of the sequence of norm of functions in Orlicz spaces which are generated by differential operators and integral operators.

\section{Notations}

Let $D=\left(D_{1}, \ldots, D_{n}\right), D_{j}=\partial / \partial x_{j}$ for $j=1,2, \ldots, n, D^{\alpha}=D_{1}^{\alpha_{1}} \ldots D_{n}^{\alpha_{n}}, \mathbf{x}^{\alpha}=x_{1}^{\alpha_{1}} \ldots x_{n}^{\alpha_{n}}$, $1 / \mathbf{x}=\left(1 / x_{1}, \ldots, 1 / x_{n}\right)$ for $\alpha=\left(\alpha_{1}, \ldots, \alpha_{n}\right) \in \mathbb{Z}_{+}^{n}, \mathbf{x}=\left(x_{1}, \ldots, x_{n}\right)$. Let $K$ be an arbitrary compact set in $\mathbb{R}^{n}, \mathbf{z} \in \mathbb{R}^{n}$ and $\varepsilon>0$. Denote by $K_{\varepsilon}:=\left\{\mathbf{x} \in \mathbb{R}^{n}: \operatorname{dist}(\mathbf{x}, K)<\varepsilon\right\}, K_{(\varepsilon)}:=$ $\left\{\mathbf{x} \in \mathbb{C}^{n}: \operatorname{dist}(\mathbf{x}, K)<\varepsilon\right\}, B(\mathbf{z}, \varepsilon):=\left\{\mathbf{x} \in \mathbb{R}^{n}:|\mathbf{x}-\mathbf{z}|<\varepsilon\right\}$ and $\left(\mathbb{R}^{n}, \varepsilon\right):=\left\{\mathbf{x} \in \mathbb{R}^{n}:\right.$ $\left.\min _{1 \leq j \leq n}\left|x_{j}\right| \geq \varepsilon\right\}$. Further, $\mathcal{S}\left(\mathbb{R}^{n}\right)$ stands for the Schwartz space on $\mathbb{R}^{n}$ and $\mathcal{S}^{\prime}\left(\mathbb{R}^{n}\right)$ is the dual space of tempered distributions on $\mathbb{R}^{n}$. The convolution of two functions $f, g$ is denoted by $f * g$. Let $f \in L^{1}\left(\mathbb{R}^{n}\right)$ then

$$
\widehat{f}(\mathbf{x})=(2 \pi)^{-n / 2} \int_{\mathbb{R}^{n}} e^{-i \mathbf{x z}} f(\mathbf{z}) d \mathbf{z},
$$

where $\mathbf{x z}=x_{1} z_{1}+x_{2} z_{2}+\cdots+x_{n} z_{n}, \mathbf{x}=\left(x_{1}, x_{2}, \ldots, x_{n}\right), \mathbf{z}=\left(z_{1}, z_{2}, \ldots, z_{n}\right)$. The Fourier transform of a tempered distribution $f$ is defined via the formula $\langle\mathcal{F} f, \phi\rangle=\langle f, \mathcal{F} \phi\rangle, \phi \in$ $\mathcal{S}\left(\mathbb{R}^{n}\right)$. Recall that (see [8]) if $f \in \mathcal{S}^{\prime}\left(\mathbb{R}^{n}\right)$ and $e_{j}=(0, \ldots, 0,1,0, \ldots, 0) \in \mathbb{Z}_{+}^{n}$ is the unit vector such that its $j^{\text {th }}$ coordinate equals $1, j=1,2, \ldots, n$, the tempered distribution $I^{e_{j}} f$ is termed a $x_{j}$-primitive of $f$ if $D^{e_{j}}\left(I^{e_{j}} f\right)=f$, that is, $\left\langle I^{e_{j}} f, D^{e_{j}} \varphi\right\rangle=-\langle f, \varphi\rangle \forall \varphi \in \mathcal{S}\left(\mathbb{R}^{n}\right)$. Now let $Q$ be a polynomial with $n$ variables, $Q(\mathbf{x}) \not \equiv 0$ and $f \in \mathcal{S}^{\prime}\left(\mathbb{R}^{n}\right)$. The tempered distribution $\mathcal{Q} f$ is termed a $Q$-primitive of $f$ if $Q(D) \mathcal{Q} f=f$. So, each $\mathcal{Q} f$ is a solution of the differential equation $Q(D) h=f$, and this is the meaning of introducing the $Q$-primitive notion. Note 
that the notion of primitives of a generalized function in $\mathcal{D}^{\prime}(a, b)$ can be found in [32]. For a polynomial $Q$, the differential operator $Q(D)$ (respectively, the integral operator $Q(I)$ ) is obtained from $Q(\mathbf{x})$ by substituting $x_{j} \rightarrow-i \partial / \partial x_{j}$ (respectively, $\left.x_{j} \rightarrow i I_{j}\right), j=1, \ldots, n$. Then, for $Q(\mathbf{x})=\sum_{|\alpha| \leq M} a_{\alpha} \mathbf{x}^{\alpha}$, we have

$$
D^{\alpha} f=\frac{\partial^{|\alpha|} f}{\partial x_{1}^{\alpha_{1}} \ldots \partial x_{n}^{\alpha_{n}}}, \quad Q(D) f=\sum_{|\alpha| \leq M} a_{\alpha}(-i)^{|\alpha|} D^{\alpha} f, \quad Q(I) f=\sum_{|\alpha| \leq M} a_{\alpha} i^{|\alpha|} I^{\alpha} f .
$$

Let $\Phi:[0,+\infty) \rightarrow[0,+\infty]$ be an arbitrary Young function, i.e., $\Phi(0)=0, \Phi(t) \geq 0$, $\Phi(t) \not \equiv 0$ and $\Phi$ is convex. Denote by $\Phi(t)=\sup _{s \geq 0}\{t s-\Phi(s)\}$ the Young function conjugate to $\Phi$ and $L^{\Phi}\left(\mathbb{R}^{n}\right)$-the space of measurable functions $u$ such that

$$
|\langle u, v\rangle|=\left|\int_{\mathbb{R}^{n}} u(\mathbf{x}) v(\mathbf{x}) d \mathbf{x}\right|<\infty
$$

for all $v$ with $\rho(v, \bar{\Phi})<\infty$, where

$$
\rho(v, \bar{\Phi})=\int_{\mathbb{R}^{n}} \bar{\Phi}(|v(\mathbf{x})|) d \mathbf{x}
$$

Then $L^{\Phi}\left(\mathbb{R}^{n}\right)$ is a Banach space with respect to the Orlicz norm

$$
\|u\|_{\Phi}=\sup _{\rho(v, \bar{\Phi}) \leq 1}\left|\int_{\mathbb{R}^{n}} u(\mathbf{x}) v(\mathbf{x}) d \mathbf{x}\right|,
$$

which is equivalent to the Luxemburg norm

$$
\|u\|_{(\Phi)}=\inf \left\{\lambda>0: \int_{\mathbb{R}^{n}} \Phi(|u(\mathbf{x})| / \lambda) d \mathbf{x} \leq 1\right\}<\infty .
$$

Moreover, $\|\cdot\|_{(\Phi)} \leq\|\cdot\|_{\Phi} \leq 2\|\cdot\|_{(\Phi)}$.

We have the following results (see [28]).

Lemma 1. Let $u \in L^{\Phi}\left(\mathbb{R}^{n}\right)$ and $v \in L^{\Phi}\left(\mathbb{R}^{n}\right)$. Then

$$
\int_{\mathbb{R}^{n}}|u(\mathbf{x}) v(\mathbf{x})| d \mathbf{x} \leq\|u\|_{\Phi}\|v\|_{(\bar{\Phi})} .
$$

Lemma 2. Let $u \in L^{\Phi}\left(\mathbb{R}^{n}\right)$ and $v \in L^{1}\left(\mathbb{R}^{n}\right)$. Then $\|u * v\|_{\Phi} \leq\|u\|_{\Phi}\|v\|_{1}$.

Note that Lebesgue spaces and their extension, Orlicz spaces, play an important role in analysis and have many applications (see $[19,20,22,24,28,29])$. Recall that $\|\cdot\|_{(\Phi)}=\|\cdot\|_{p}$, where $\Phi(t)=t^{p}$ with $1 \leq p<\infty$, and $\|\cdot\|_{(\Phi)}=\|\cdot\|_{\infty}$, where $\Phi(t)=0$ for $0 \leq t \leq 1$ and $\Phi(t)=\infty$ for $t>1$.

\section{Some spectral formulas for $Q$-primitives of a function}

Theorem 1. Let $\Phi$ be an arbitrary Young function, $f \in L^{\Phi}\left(\mathbb{R}^{n}\right)$ and $Q$ be a polynomial. Assume that $\mathrm{sp}(f)$ is compact and $Q(\mathbf{x}) \neq 0$ for all $\mathbf{x} \in \mathrm{sp}(f)$. Then there exists exactly one sequence of functions $\left(\mathcal{Q}^{m} f\right)_{m=0}^{\infty} \subset L^{\Phi}\left(\mathbb{R}^{n}\right)$ satisfying $\mathcal{Q}^{0} f=f, Q(D) \mathcal{Q}^{m+1} f=\mathcal{Q}^{m} f, \operatorname{sp}(f)=\operatorname{sp}\left(\mathcal{Q}^{m} f\right)$ for $m \in \mathbb{Z}_{+}$. 
Proof. We consider a function $\varphi \in C_{0}^{\infty}\left(\mathbb{R}^{n}\right)$ satisfying $\varphi(\mathbf{x})=1$ for $\mathbf{x}$ in a neighborhood of $\operatorname{sp}(f)$. Put $\mathcal{Q}^{m} f=(2 \pi)^{-n / 2} f * \mathcal{F}^{-1}\left(\varphi(\mathbf{x}) / Q^{m}(\mathbf{x})\right)$. Clearly, $\mathcal{Q}^{0} f=f, \mathcal{Q}^{m} f \in L^{\Phi}\left(\mathbb{R}^{n}\right)$, $\operatorname{sp}(f)=\operatorname{sp}\left(\mathcal{Q}^{m} f\right)$ and

$$
Q(D) \mathcal{Q}^{m+1} f=(2 \pi)^{-n / 2} f *\left(Q(D)\left(\mathcal{F}^{-1}(\varphi(\mathbf{x})) / Q^{m+1}(\mathbf{x})\right)\right)=\mathcal{Q}^{m} f
$$

for all $m=0,1, \ldots$ Moreover, $\mathcal{Q}_{1} f$ and $\mathcal{Q}_{2} f$ are two $Q$-primitives in $L^{\Phi}\left(\mathbb{R}^{n}\right)$ of $f$ satisfying $\operatorname{sp}(f)=\operatorname{sp}\left(\mathcal{Q}_{1} f\right)=\operatorname{sp}\left(\mathcal{Q}_{2} f\right)$ then

$$
\begin{aligned}
\langle f, \mathcal{F}(\varphi(\mathbf{x}) \psi(\mathbf{x}) / Q(\mathbf{x}))\rangle & =\left\langle Q(D) \mathcal{Q}_{j} f, \mathcal{F}(\varphi(\mathbf{x}) \psi(\mathbf{x}) / Q(\mathbf{x}))\right\rangle \\
& =\left\langle\mathcal{Q}_{j} f, Q(-D) \mathcal{F}(\varphi(\mathbf{x}) \psi(\mathbf{x}) / Q(\mathbf{x}))\right\rangle=\left\langle\mathcal{Q}_{j} f, \mathcal{F}(\varphi(\mathbf{x}) \psi(\mathbf{x})\rangle\right. \\
& =\left\langle\mathcal{F}\left(\mathcal{Q}_{j} f\right), \varphi \psi\right\rangle=\left\langle\mathcal{F}\left(\mathcal{Q}_{j} f\right), \psi\right\rangle=\left\langle\mathcal{Q}_{j} f, \mathcal{F} \psi\right\rangle
\end{aligned}
$$

for $j=1,2$ and $\psi \in \mathcal{S}\left(\mathbb{R}^{n}\right)$. Hence, $\left\langle\mathcal{Q}_{1} f-\mathcal{Q}_{2} f, \mathcal{F} \psi\right\rangle=0$ for all $\psi \in \mathcal{S}\left(\mathbb{R}^{n}\right)$, and then $\mathcal{Q}_{1} f=\mathcal{Q}_{2} f$ because of $\mathcal{F}\left(\mathcal{S}\left(\mathbb{R}^{n}\right)\right)=\mathcal{S}\left(\mathbb{R}^{n}\right)$, so the uniqueness of the sequence $\left(\mathcal{Q}^{m} f\right)_{m=0}^{\infty}$ is proved.

Remark 1. Let $f \in L^{\Phi}\left(\mathbb{R}^{n}\right)$ and $\operatorname{sp}(f)$ be compact. It should be noticed that the assumption $Q(\mathbf{x}) \neq 0 \forall \mathbf{x} \in \operatorname{sp}(f)$ is essential for the existence of $Q$-primitives in $L^{\Phi}\left(\mathbb{R}^{n}\right)$ of $f$. For example, if $f(\mathbf{x})=1+\cos \mathbf{x}, Q(\mathbf{x})=\mathbf{x}, n=1, \Phi(\mathbf{x})=0$ for $0 \leq \mathbf{x} \leq 1$ and $\Phi(\mathbf{x})=\infty$ for $\mathbf{x}>1$, then $\operatorname{sp}(f)=\{-1,0,1\}$ and each $Q$-primitive of $f$ has the form $\mathbf{x}+\sin \mathbf{x}+c, c \in \mathbb{C}$, which does not belong to $L^{\Phi}(\mathbb{R})\left(=L^{\infty}(\mathbb{R})\right)$.

Theorem 2. Let $\Phi$ be an arbitrary Young function, $Q$ be a polynomial and $\left(\mathcal{Q}^{m} f\right)_{m=0}^{\infty} \subset L^{\Phi}\left(\mathbb{R}^{n}\right)$ satisfies $\mathcal{Q}^{0} f=f, Q(D) \mathcal{Q}^{m+1} f=\mathcal{Q}^{m} f$ for $m \in \mathbb{Z}_{+}$. Then

$$
\liminf _{m \rightarrow \infty}\left\|\mathcal{Q}^{m} f\right\|_{\Phi}^{1 / m} \geq \sup _{\mathbf{x} \in \operatorname{sp}(f)}|1 / Q(\mathbf{x})|
$$

Before giving the proof of above theorem, we recall the following result in [7].

Lemma 3. Let $\Phi$ be an arbitrary Young function, $Q$ be a polynomial, $f \in L^{\Phi}\left(\mathbb{R}^{n}\right)$ and $s p(f)$ be compact. Then

$$
\lim _{m \rightarrow \infty}\left\|Q^{m}(D) f\right\|_{\Phi}^{1 / m}=\sup \{|Q(\mathbf{x})|: \mathbf{x} \in \operatorname{sp}(f)\}
$$

Proof of Theorem 2. Given $\varrho \in \operatorname{sp}(f)$. Then for any $\varepsilon>0$ there exists $\psi \in C_{0}^{\infty}\left(\mathbb{R}^{n}\right)$, supp $\psi \subset$ $B(\varrho, \varepsilon)$ such that $\langle\widehat{f}, \psi\rangle \neq 0$. Put $\mathcal{H}_{m}:=\mathcal{F}\left(\psi(\mathbf{x}) Q^{m}(\mathbf{x})\right)$. Clearly, $\mathcal{H}_{m} \in \mathcal{S}\left(\mathbb{R}^{n}\right)$, and

$$
\langle\widehat{f}, \psi\rangle=\langle f, \mathcal{F} \psi\rangle=\left\langle Q^{m}(D) \mathcal{Q}^{m} f, \mathcal{F} \psi\right\rangle=\left\langle\mathcal{Q}^{m} f, Q^{m}(-D) \mathcal{F} \psi\right\rangle=\left\langle\mathcal{Q}^{m} f, \mathcal{H}_{m}\right\rangle .
$$

Using Lemma 1, we get

$$
\left\|\mathcal{Q}^{m} f\right\|_{\Phi}\left\|\mathcal{H}_{m}\right\|_{(\bar{\Phi})} \geq\left|\left\langle\mathcal{Q}^{m} f, \mathcal{H}_{m}\right\rangle\right|=|\langle\widehat{f}, \psi\rangle|>0 .
$$

Hence,

$$
\liminf _{m \rightarrow \infty}\left\|\mathcal{Q}^{m} f\right\|_{\Phi}^{1 / m} \geq 1 / \limsup _{m \rightarrow \infty}\left\|\mathcal{H}_{m}\right\|_{(\bar{\Phi})}^{1 / m}
$$


From Lemma 3, we deduce

$$
\limsup _{m \rightarrow \infty}\left\|\mathcal{H}_{m}\right\|_{(\bar{\Phi})}^{1 / m} \leq \sup _{\mathbf{x} \in B(\varrho, \varepsilon)}|Q(\mathbf{x})|
$$

Therefore, since (3),

$$
\liminf _{m \rightarrow \infty}\left\|\mathcal{Q}^{m} f\right\|_{\Phi}^{1 / m} \geq 1 / \sup _{\mathbf{x} \in B(\varrho, \varepsilon)}|Q(\mathbf{x})|
$$

Letting $\varepsilon \rightarrow 0$, we get

$$
\liminf _{m \rightarrow \infty}\left\|\mathcal{Q}^{m} f\right\|_{\Phi}^{1 / m} \geq 1 /|Q(\varrho)| .
$$

Because (4) holds for any $\varrho \in \operatorname{sp}(f)$, we confirm (1), which completes the proof.

We have the following theorem for entire functions of exponential type.

Theorem 3. Let $\Phi$ be an arbitrary Young function, $Q$ be a polynomial and $\left(\mathcal{Q}^{m} f\right)_{m=0}^{\infty} \subset L^{\Phi}\left(\mathbb{R}^{n}\right)$ satisfies $\mathcal{Q}^{0} f=f, Q(D) \mathcal{Q}^{m+1} f=\mathcal{Q}^{m} f$ for $m \in \mathbb{Z}_{+}$. Assume that $\operatorname{sp}(f)$ is compact and $\operatorname{sp}\left(\mathcal{Q}^{m} f\right)=\operatorname{sp}(f)$ for all $m \in \mathbb{Z}_{+}$. Then

$$
\lim _{m \rightarrow \infty}\left\|\mathcal{Q}^{m} f\right\|_{\Phi}^{1 / m}=\sup _{\mathbf{x} \in \operatorname{sp}(f)}|1 / Q(\mathbf{x})|
$$

Proof. We divide the proof into two cases.

Case $1(Q(\mathbf{x}) \neq 0 \forall \mathbf{x} \in \operatorname{sp}(f))$. Put $K:=\mathrm{sp}(f)$. Now we prove

$$
\limsup _{m \rightarrow \infty}\left\|\mathcal{Q}^{m} f\right\|_{\Phi}^{1 / m} \leq \sup _{\mathbf{x} \in K}|1 / Q(\mathbf{x})|
$$

Indeed, by virtue of $Q(\mathbf{x}) \neq 0 \forall \mathbf{x} \in K$ there exists a small number $\varepsilon>0$ such that $Q(\mathbf{x}) \neq 0$ $\forall \mathbf{x} \in K_{\varepsilon}$. We choose a function $\mathcal{J} \in C_{0}^{\infty}\left(\mathbb{R}^{n}\right): \mathcal{J}(\mathbf{x})=1$ if $\mathbf{x} \in K_{\varepsilon / 2}$ and $\mathcal{J}(\mathbf{x})=0$ if $\mathbf{x} \notin K_{\varepsilon}$. Then $\mathcal{J}(\mathbf{x}) / Q^{m}(\mathbf{x}) \in \mathcal{S}\left(\mathbb{R}^{n}\right)$. Because of $Q^{m}(D) \mathcal{Q}^{m} f=f$ and $\operatorname{sp}\left(\mathcal{Q}^{m} f\right)=\operatorname{sp}(f)$, we have $\widehat{f} \mathcal{J}(\mathbf{x}) / Q^{m}(\mathbf{x})=\widehat{\mathcal{Q}^{m} f}$. Consequently, $\mathcal{Q}^{m} f=(2 \pi)^{-n / 2} f * \mathcal{F}^{-1}\left(\mathcal{J}(\mathbf{x}) / Q^{m}(\mathbf{x})\right)$.

Hence, using Lemma 2, we obtain

$$
\left\|\mathcal{Q}^{m} f\right\|_{\Phi} \leq(2 \pi)^{-n / 2}\left\|\mathcal{F}^{-1}\left(\mathcal{J}(\mathbf{x}) / Q^{m}(\mathbf{x})\right)\right\|_{1}\|f\|_{\Phi}=(2 \pi)^{-n / 2}\left\|\mathcal{F}\left(\mathcal{J}(\mathbf{x}) / Q^{m}(\mathbf{x})\right)\right\|_{1} \mid f \|_{\Phi} .
$$

So,

$$
\limsup _{m \rightarrow \infty}\left\|\mathcal{Q}^{m} f\right\|_{\Phi}^{1 / m} \leq \limsup _{m \rightarrow \infty}\left\|\mathcal{F}\left(\mathcal{J}(\mathbf{x}) / Q^{m}(\mathbf{x})\right)\right\|_{1}^{1 / m} .
$$

We define the function $\mathcal{G}_{m}$ as follows $\mathcal{G}_{m}=\mathcal{F}\left(\mathcal{J}(\mathbf{x}) / Q^{m}(\mathbf{x})\right)$. Then for $\sigma \in \mathbb{Z}_{+}^{n}, \sigma \leq(2,2, \ldots, 2)$ we have the following estimate

$$
\begin{aligned}
\sup _{\mathbf{y} \in \mathbb{R}^{n}}\left|\mathbf{y}^{\sigma} \mathcal{G}_{m}(\mathbf{y})\right| & \leq(2 \pi)^{-n / 2} \sup _{\mathbf{y} \in \mathbb{R}^{n}}\left|\int_{\mathbb{R}^{n}} e^{-i \mathbf{y x}} D^{\sigma}\left(\mathcal{J}(\mathbf{x}) / Q^{m}(\mathbf{x})\right) d \mathbf{x}\right| \\
& =(2 \pi)^{-n / 2} \sup _{\mathbf{y} \in \mathbb{R}^{n}}\left|\int_{K_{\varepsilon}} e^{-i \mathbf{y x}} D^{\sigma}\left(\mathcal{J}(\mathbf{x}) / Q^{m}(\mathbf{x})\right) d \mathbf{x}\right| \\
& \leq(2 \pi)^{-n / 2} \int_{K_{\varepsilon}}\left|D^{\sigma}\left(\mathcal{J}(\mathbf{x}) / Q^{m}(\mathbf{x})\right)\right| d \mathbf{x},
\end{aligned}
$$


which together with Leibniz's rule imply

$$
\begin{aligned}
\sup _{\mathbf{y} \in \mathbb{R}^{n}}\left|\mathbf{y}^{\sigma} \mathcal{G}_{m}(\mathbf{y})\right| & \leq(2 \pi)^{-n / 2} \int_{K_{\varepsilon}}\left|\sum_{\gamma \leq \sigma} \frac{\sigma !}{\gamma !(\sigma-\gamma) !} D^{\gamma} \mathcal{J}(\mathbf{x}) D^{\sigma-\gamma}\left(1 / Q^{m}(\mathbf{x})\right)\right| d \mathbf{x} \\
& \leq(2 \pi)^{-n / 2} \sum_{\gamma \leq \sigma}\left(\frac{\sigma !}{\gamma !(\sigma-\gamma) !} \sup _{\mathbf{y} \in K_{\varepsilon}}\left|D^{\sigma-\gamma}\left(1 / Q^{m}(\mathbf{y})\right)\right| \int_{K_{\varepsilon}}\left|D^{\gamma} \mathcal{J}(\mathbf{x})\right| d \mathbf{x}\right) \\
& \leq(2 \pi)^{-n / 2} \max _{\tau \leq(2,2, \ldots, 2)} \sup _{\mathbf{y} \in K_{\varepsilon}}\left|D^{\tau}\left(1 / Q^{m}(\mathbf{y})\right)\right| \sum_{\gamma \leq \sigma}\left(\frac{\sigma !}{\gamma !(\sigma-\gamma) !} \int_{K_{\varepsilon}}\left|D^{\gamma} \mathcal{J}(\mathbf{x})\right| d \mathbf{x}\right) .
\end{aligned}
$$

From

$$
D^{\tau}\left(1 / Q^{m}(\mathbf{y})\right)=\sum_{\substack{\kappa \in \mathbb{Z}_{+},\left(\kappa_{j}\right)_{j=1}^{n} \subset \mathbb{Z}_{+,}^{n} \\ \kappa \leq 2 n, \kappa_{j} \leq(2,2, \ldots, 2)}} c_{\kappa,\left(\kappa_{j}\right)_{j=1}^{n}}(m-1+\kappa) !\left(\prod_{j=1}^{n} D^{\kappa_{j}} Q(\mathbf{y})\right) /\left((m-1) ! Q^{m+\kappa}(\mathbf{y})\right)
$$

and $\inf \left\{|Q(\mathbf{y})|: \mathbf{y} \in K_{\varepsilon}\right\}>0$, there is a constant $C<\infty$ independent of $m$ such that

$$
\sup _{\mathbf{y} \in K_{\varepsilon}}\left|D^{\tau}\left(1 / Q^{m}(\mathbf{y})\right)\right| \leq C m^{2 n} \sup _{\mathbf{y} \in K_{\varepsilon}}\left|1 / Q^{m}(\mathbf{y})\right|, \quad \forall \tau \in \mathbb{Z}_{+}^{n}, \quad \tau \leq(2,2, \ldots, 2) .
$$

Combining (7) and (8), we have

$$
\begin{aligned}
\sup _{\mathbf{y} \in \mathbb{R}^{n}}\left|\mathbf{y}^{\sigma} \mathcal{G}_{m}(\mathbf{y})\right| & \leq(2 \pi)^{-n / 2} C m^{2 n} \sup _{\mathbf{y} \in K_{\varepsilon}}\left|1 / Q^{m}(\mathbf{y})\right| \sum_{\gamma \leq \sigma}\left(\frac{\sigma !}{\gamma !(\sigma-\gamma) !} \int_{K_{\varepsilon}}\left|D^{\gamma} \mathcal{J}(\mathbf{x})\right| d \mathbf{x}\right) \\
& =C_{1} m^{2 n} \sup _{\mathbf{y} \in K_{\varepsilon}}|1 / Q(\mathbf{y})|^{m},
\end{aligned}
$$

where

$$
C_{1}:=C(2 \pi)^{-n / 2} \sum_{\gamma \leq \sigma}\left(\frac{\sigma !}{\gamma !(\sigma-\gamma) !} \int_{K_{\varepsilon}}\left|D^{\gamma} \mathcal{J}(\mathbf{x})\right| d \mathbf{x}\right) .
$$

Clearly, $C_{1}$ is independent of $m$. Then it follows from (9) and

$$
\begin{aligned}
\left\|\mathcal{G}_{m}\right\|_{1} & \leq\left(\sup _{\mathbf{y} \in \mathbb{R}^{n}} \mid\left(1+y_{1}^{2}\right)\left(1+y_{2}^{2}\right) \ldots\left(1+y_{n}^{2}\right) \mathcal{G}_{m}(\mathbf{y})\right)\left(\int_{\mathbb{R}^{n}} \frac{d \mathbf{y}}{\left(1+y_{1}^{2}\right)\left(1+y_{2}^{2}\right) \ldots\left(1+y_{n}^{2}\right)}\right) \\
& =\pi^{n} \sup _{\mathbf{y} \in \mathbb{R}^{n}}\left|\left(1+y_{1}^{2}\right)\left(1+y_{2}^{2}\right) \ldots\left(1+y_{n}^{2}\right) \mathcal{G}_{m}(\mathbf{y})\right|
\end{aligned}
$$

that

$$
\limsup _{m \rightarrow \infty}\left\|\mathcal{G}_{m}\right\|_{1}^{1 / m} \leq \sup _{\mathbf{y} \in K_{\varepsilon}}|1 / Q(\mathbf{y})|
$$

From (6) and (10), we obtain $\limsup _{m \rightarrow \infty}\left\|\mathcal{Q}^{m} f\right\|_{\Phi}^{1 / m} \leq \sup _{\mathbf{y} \in K_{\varepsilon}}|1 / Q(\mathbf{y})|$. Letting $\varepsilon \rightarrow 0$, we confirm (5). By Theorem 2 and (5), we get $\lim _{m \rightarrow \infty}\left\|\mathcal{Q}^{m} f\right\|_{\Phi}^{1 / m}=\sup _{\mathbf{y} \in \operatorname{sp}(f)}|1 / Q(\mathbf{y})|$.

Case $2(Q(\mathbf{x})=0$ for some $\mathbf{x} \in \operatorname{sp}(f))$. Then it follows from Theorem 2 that

$$
\liminf _{m \rightarrow \infty}\left\|\mathcal{Q}^{m} f\right\|_{\Phi}^{1 / m}=\infty
$$


Remark 2. Note that due to Theorem 1, the assumption $\operatorname{sp}\left(\mathcal{Q}^{m} f\right)=\operatorname{sp}(f)$ for all $m \in \mathbb{Z}_{+}$may be replaced by the following stricter condition: $Q(\mathbf{x}) \neq 0$ for all $\mathbf{x} \in \operatorname{sp}(f)$.

Remark 3. In general, it is impossible to calculate all $\left\|\mathcal{Q}^{m} f\right\|_{\Phi}, m=1,2, \ldots$, while Theorems 2 and 3 give us asymptotic estimations of them by using the following rather easier calculation of $\sup _{\mathbf{x} \in \operatorname{sp}(f)}|1 / Q(\mathbf{x})|$, which is especially effect if $f \in \mathcal{F}^{-1}(\mathcal{M})$, where $\mathcal{M}$ is the set of all measurable functions belonging to $\mathcal{S}^{\prime}\left(\mathbb{R}^{n}\right)$. The similar situation occurs with our theorems obtained in the following sections. This shows that our results have the potential to apply to computational science.

\section{Some spectral formulas for functions generated by integral operators}

Let $\Phi$ be an arbitrary Young function and $f \in L^{\Phi}\left(\mathbb{R}^{n}\right)$. If $\operatorname{sp}(f) \subset\left(\mathbb{R}^{n}, v\right)$ for some $v>0$, then it was shown in [10] that there exists exactly one sequence $\left(I^{\alpha} f\right)_{\alpha \in \mathbb{Z}_{+}^{n}} \subset L^{\Phi}\left(\mathbb{R}^{n}\right)$ satisfying $D^{\alpha} I^{\alpha+\ell} f=I^{\ell} f$ for all $\alpha, \ell \in \mathbb{Z}_{+}^{n}$.

Theorem 4. Let $\Phi$ be an arbitrary Young function, $Q$ be a polynomial and $\left(I^{\alpha} f\right)_{\alpha \in \mathbb{Z}_{+}^{n}} \subset L^{\Phi}\left(\mathbb{R}^{n}\right)$ satisfying $D^{\alpha} I^{\alpha+\ell} f=I^{\ell} f$ for all $\alpha, \ell \in \mathbb{Z}_{+}^{n}$. Assume that $\operatorname{sp}(f) \subset\left(\mathbb{R}^{n}, v\right)$ for some $v>0$. Then $\operatorname{sp}\left(Q^{m}(I) f\right) \subset \operatorname{sp}(f) \forall m \in \mathbb{Z}_{+}$, and

$$
\liminf _{m \rightarrow \infty}\left\|Q^{m}(I) f\right\|_{\Phi}^{1 / m} \geq \sup _{\mathbf{x} \in \operatorname{sp}(f)}|Q(1 / \mathbf{x})|
$$

Proof. It was shown in [10] that $\operatorname{sp}\left(I^{\alpha} f\right)=\operatorname{sp}(f) \forall \alpha \in \mathbb{Z}_{+}^{n}$. Hence, $\operatorname{sp}\left(Q^{m}(I) f\right) \subset \operatorname{sp}(f)$ $\forall m \in \mathbb{Z}_{+}$. Next, we show that

$$
\liminf _{m \rightarrow \infty}\left\|Q^{m}(I) f\right\|_{\Phi}^{1 / m} \geq|Q(1 / \varrho)|
$$

where $\varrho$ is an arbitrary element in $\operatorname{sp}(f)$. Indeed, if $Q(1 / \varrho)=0$, then (11) is obvious. If $Q(1 / \varrho) \neq 0$, then for a small enough number $\varepsilon \in(0, v / 2)$ we have $\prod_{j=1}^{n}\left|x_{j}\right|>0$ and $Q(1 / \mathbf{x}) \neq 0 \forall \mathbf{x} \in B(\varrho, \varepsilon)$. From $\varrho \in \operatorname{sp}(f)$, there is $\mathcal{J} \in C_{0}^{\infty}\left(\mathbb{R}^{n}\right)$, supp $\mathcal{J} \subset B(\varrho, \varepsilon)$ such that $\langle\widehat{f}, \mathcal{J}\rangle \neq 0$. Put $\mathcal{H}_{m}=\mathcal{F}\left(\mathcal{J}(\mathbf{x}) / Q^{m}(1 / \mathbf{x})\right)$. Clearly, $\mathcal{H}_{m}$ is well defined, $\mathcal{H}_{m} \in \mathcal{S}\left(\mathbb{R}^{n}\right)$ and it follows from $f=D^{\alpha} I^{\alpha} f$ that

$$
\begin{aligned}
\left\langle f, \mathcal{F}\left(\mathcal{J}(\mathbf{x}) /\left((i \mathbf{x})^{\alpha} Q^{m}(1 / \mathbf{x})\right)\right)\right\rangle & =\left\langle D^{\alpha} I^{\alpha} f, \mathcal{F}\left(\mathcal{J}(\mathbf{x}) /\left((i \mathbf{x})^{\alpha} Q^{m}(1 / \mathbf{x})\right)\right)\right\rangle \\
& =(-1)^{|\alpha|}\left\langle I^{\alpha} f, D^{\alpha} \mathcal{F}\left(\mathcal{J}(\mathbf{x}) /\left((i \mathbf{x})^{\alpha} Q^{m}(1 / \mathbf{x})\right)\right)\right\rangle=\left\langle I^{\alpha} f, \mathcal{H}_{m}\right\rangle
\end{aligned}
$$

for all $\alpha \in \mathbb{Z}_{+}^{n}$. Then, for $Q^{m}(\mathbf{x})=\sum_{\alpha} c_{\alpha} \mathbf{x}^{\alpha}$, we get

$$
\begin{aligned}
\left\langle Q^{m}(I) f, \mathcal{H}_{m}\right\rangle & =\sum_{\alpha} c_{\alpha} i^{|\alpha|}\left\langle I^{\alpha} f, \mathcal{H}_{m}\right\rangle=\sum_{\alpha} c_{\alpha} i^{|\alpha|}\left\langle f, \mathcal{F}\left(\mathcal{J}(\mathbf{x}) /\left((i \mathbf{x})^{\alpha} Q^{m}(1 / \mathbf{x})\right)\right)\right\rangle \\
& =\left\langle f, \mathcal{F}\left(\mathcal{J}(\mathbf{x}) \sum_{\alpha} c_{\alpha}(1 / \mathbf{x})^{\alpha} /\left(Q^{m}(1 / \mathbf{x})\right)\right)\right\rangle=\langle f, \widehat{\mathcal{J}}\rangle=\langle\widehat{f}, \mathcal{J}\rangle .
\end{aligned}
$$

So, using Lemma 1, we have

$$
0<|\langle\widehat{f}, \mathcal{J}\rangle|=\left|\left\langle Q^{m}(I) f, \mathcal{H}_{m}\right\rangle\right| \leq\left\|Q^{m}(I) f\right\|_{\Phi}\left\|\mathcal{H}_{m}\right\|_{(\Phi)}
$$

It follows that

$$
\liminf _{m \rightarrow \infty}\left\|Q^{m}(I) f\right\|_{\Phi}^{1 / m} \geq 1 / \limsup _{m \rightarrow \infty}\left\|\mathcal{H}_{m}\right\|_{(\bar{\Phi})}^{1 / m}
$$


Arguing as in the proof of Theorem 3 and taking account of $B(\varrho, \varepsilon) \subset\left(\mathbb{R}^{n}, v / 2\right)$, we have a constant $A<\infty$ independent of $m, \sigma$ such that

$$
\sup _{\mathbf{z} \in \mathbb{R}^{n}}\left|\mathbf{z}^{\sigma} \mathcal{H}_{m}(\mathbf{z})\right| \leq A m^{2 n} \sup _{\mathbf{z} \in B(\varrho, \varepsilon)}\left|1 / Q^{m}(1 / \mathbf{z})\right|,
$$

for all $\sigma \in \mathbb{Z}_{+}^{n}, \sigma \leq(2,2, \ldots, 2)$. Consequently,

$$
\sup _{\mathbf{z} \in \mathbb{R}^{n}}\left|\left(1+z_{1}^{2}\right)\left(1+z_{2}^{2}\right) \ldots\left(1+z_{n}^{2}\right) \mathcal{H}_{m}(\mathbf{z})\right| \leq A 2^{n} m^{2 n} \sup _{\mathbf{z} \in B(\varrho, \varepsilon)}\left|1 / Q^{m}(1 / \mathbf{z})\right| .
$$

Moreover,

$$
\left\|\mathcal{H}_{m}\right\|_{(\bar{\Phi})} \leq\|\mathrm{Y}\|_{(\bar{\Phi})} \sup _{\mathbf{z} \in \mathbb{R}^{n}}\left|\left(1+z_{1}^{2}\right)\left(1+z_{2}^{2}\right) \ldots\left(1+z_{n}^{2}\right) \mathcal{H}_{m}(\mathbf{z})\right|
$$

where

$$
\mathrm{Y}(\mathbf{z})=\frac{1}{\left(1+z_{1}^{2}\right)\left(1+z_{2}^{2}\right) \ldots\left(1+z_{n}^{2}\right)} .
$$

We choose $\kappa>0$ such that $\bar{\Phi}(\kappa)<\infty$. Since $\bar{\Phi}$ is a Young function, $\bar{\Phi}(x) / x$ is increasing on $[0,+\infty)$. Then $\bar{\Phi}(x) \leq \kappa_{1} x$ for all $x \in[0, \kappa]$, where $\kappa_{1}=\bar{\Phi}(\kappa) / \kappa$. Hence,

$$
\int_{\mathbb{R}^{n}} \Phi\left(\frac{1}{\lambda\left(\prod_{j=1}^{n}\left(1+z_{j}^{2}\right)\right)}\right) d \mathbf{z} \leq \int_{\mathbb{R}^{n}} \frac{\kappa_{1}}{\lambda\left(\prod_{j=1}^{n}\left(1+z_{j}^{2}\right)\right)} d \mathbf{z}=\frac{\kappa_{1} \pi^{n}}{\lambda}<1
$$

for all $\lambda>\max \left\{1 / \kappa, \kappa_{1} \pi^{n}\right\}$. Consequently, $\|\mathrm{Y}\|_{(\bar{\Phi})} \leq \max \left\{1 / \kappa, \kappa_{1} \pi^{n}\right\}<\infty$, which together with (13) and (14) imply

$$
\limsup _{m \rightarrow \infty}\left\|\mathcal{H}_{m}\right\|_{(\bar{\Phi})}^{1 / m} \leq \sup _{\mathbf{z} \in B(\varrho, \varepsilon)} 1 /|Q(1 / \mathbf{z})|
$$

Therefore, since (12), $\liminf _{m \rightarrow \infty}\left\|Q^{m}(I) f\right\|_{\Phi}^{1 / m} \geq \inf _{\mathbf{z} \in B(\varrho, \varepsilon)}|Q(1 / \mathbf{z})|$. Letting $\varepsilon \rightarrow 0$, we confirm (11). Because (11) holds for any $\varrho \in \operatorname{sp}(f)$, we obtain

$$
\liminf _{m \rightarrow \infty}\left\|Q^{m}(I) f\right\|_{\Phi}^{1 / m} \geq \sup _{\mathbf{x} \in \operatorname{sp}(f)}|Q(1 / \mathbf{x})|
$$

We have the following theorem for entire functions of exponential type.

Theorem 5. Let $\Phi$ be an arbitrary Young function, $Q$ be a polynomial and $\left(I^{\alpha} f\right)_{\alpha \in \mathbb{Z}_{+}^{n}} \subset L^{\Phi}\left(\mathbb{R}^{n}\right)$ satisfying $D^{\alpha} I^{\alpha+\sigma} f=I^{\sigma} f$ for all $\alpha, \sigma \in \mathbb{Z}_{+}^{n}$. Assume that $\operatorname{sp}(f)$ is compact and $\operatorname{sp}(f) \subset\left(\mathbb{R}^{n}, v\right)$ for some $v>0$. Then

$$
\lim _{m \rightarrow \infty}\left\|Q^{m}(I) f\right\|_{\Phi}^{1 / m}=\sup _{\mathbf{x} \in \operatorname{sp}(f)}|Q(1 / \mathbf{x})| .
$$

Proof. We put $K=\operatorname{sp}(f)$ and consider $\varepsilon \in(0, v)$. Then there exists $\mathcal{J} \in C_{0}^{\infty}\left(\mathbb{R}^{n}\right)$ such that $\mathcal{J}(\mathbf{x})=1$ if $\mathbf{x} \in K_{\varepsilon / 2}$ and $\mathcal{J}(\mathbf{x})=0$ if $\mathbf{x} \notin K_{\varepsilon}$. So, supp $\mathcal{J} \subset\left(\mathbb{R}^{n}, v-\varepsilon\right)$. From $\widehat{f}=(i \mathbf{x})^{\alpha} \widehat{I^{\alpha} f}$, we get $\mathcal{J}(\mathbf{x}) \widehat{f}=(i \mathbf{x})^{\alpha} \bar{I}^{\alpha} f$ and then it follows from $\operatorname{sp}\left(I^{\alpha} f\right)=\operatorname{sp}(f)$ that $\widehat{f} \mathcal{J}(\mathbf{x}) /(i \mathbf{x})^{\alpha}=\widehat{I^{\alpha} f}$ for all $\alpha \in \mathbb{Z}_{+}^{n}$. Therefore, $\widehat{Q^{m}(I)} f=\widehat{f} \mathcal{J}(\mathbf{x}) Q^{m}(1 / \mathbf{x})$ for all $\forall m \in \mathbb{Z}_{+}$. So,

$$
Q^{m}(I) f=(2 \pi)^{-n / 2} f * \mathcal{F}^{-1}\left(\mathcal{J}(\mathbf{x}) Q^{m}(1 / \mathbf{x})\right) \quad \forall m \in \mathbb{Z}_{+} .
$$


Then, using Lemma 2, we get

$$
\left\|Q^{m}(I) f\right\|_{\Phi} \leq(2 \pi)^{-n / 2}\|f\|_{\Phi}\left\|\mathcal{F}^{-1}\left(\mathcal{J}(\mathbf{x}) Q^{m}(1 / \mathbf{x})\right)\right\|_{1} \quad \forall m \in \mathbb{Z}_{+} .
$$

Thus, $\limsup _{m \rightarrow \infty}\left\|Q^{m}(I) f\right\|_{\Phi}^{1 / m} \leq \limsup _{m \rightarrow \infty}\left\|\mathcal{G}_{m}\right\|_{1}^{1 / m}$, where $\mathcal{G}_{m}:=\mathcal{F}^{-1}\left(\mathcal{J}(\mathbf{x}) Q^{m}(1 / \mathbf{x})\right)$. Then it follows from $\left\|\mathcal{G}_{m}\right\|_{1} \leq \pi^{n} \sup _{\mathbf{z} \in \mathbb{R}^{n}}\left|\left(1+z_{1}^{2}\right)\left(1+z_{2}^{2}\right) \ldots\left(1+z_{n}^{2}\right) \mathcal{G}_{m}(\mathbf{z})\right|$ that

$$
\limsup _{m \rightarrow \infty}\left\|Q^{m}(I) f\right\|_{\Phi}^{1 / m} \leq \limsup _{m \rightarrow \infty} \sup _{\mathbf{z} \in \mathbb{R}^{n}}\left|\left(1+z_{1}^{2}\right)\left(1+z_{2}^{2}\right) \ldots\left(1+z_{n}^{2}\right) \mathcal{G}_{m}(\mathbf{z})\right|^{1 / m} .
$$

Arguing as in the proof of Theorem 3, we have a constant $B<\infty$ independent of $m, \sigma$ such that

$$
\sup _{\mathbf{z} \in \mathbb{R}^{n}}\left|\mathbf{z}^{\sigma} \mathcal{G}_{m}(\mathbf{z})\right| \leq B m^{2 n} \sup _{\mathbf{z} \in K_{\varepsilon}}\left|Q^{m}(1 / \mathbf{z})\right|,
$$

for all $\sigma \in \mathbb{Z}_{+}^{n}, \sigma \leq(2,2, \ldots, 2)$. Combining (15) and (16), we obtain

$$
\limsup _{m \rightarrow \infty}\left\|Q^{m}(I) f\right\|_{\Phi}^{1 / m} \leq \sup _{\mathbf{z} \in K_{\varepsilon}}|Q(1 / \mathbf{z})| .
$$

Letting $\varepsilon \rightarrow 0$, we deduce

$$
\limsup _{m \rightarrow \infty}\left\|Q^{m}(I) f\right\|_{\Phi}^{1 / m} \leq \sup _{\mathbf{x} \in K}|Q(1 / \mathbf{x})|
$$

Combining (17) and Theorem 4, we arrive

$$
\lim _{m \rightarrow \infty}\left\|Q^{m}(I) f\right\|_{\Phi}^{1 / m}=\sup _{\mathbf{x} \in \operatorname{sp}(f)}|Q(1 / \mathbf{x})|
$$

\section{Some spectral formulas for functions generated by differential operators}

If $f \in \mathcal{S}^{\prime}\left(\mathbb{R}^{n}\right)$ has compact spectrum, then $f$ is the Fourier transform of $v:=\mathcal{F}^{-1} f$. The Fourier-Laplace transform of $v$ (see [32]), still denoted by the same symbol $f$, is known as follows $f(\zeta)=(2 \pi)^{-n / 2}\left\langle v(\cdot), e^{-i \zeta \cdot}\right\rangle, \zeta \in \mathbb{C}^{n}$. This is an entire function on $\mathbb{C}^{n}$. Hence, for $f \in L^{\Phi}\left(\mathbb{R}^{n}\right)$ and $\mathbf{a} \in \mathbb{R}^{n}$ (or $f \in \mathcal{F}\left(\mathcal{E}^{\prime}\left(\mathbb{R}^{n}\right)\right)$ and $\left.\mathbf{a} \in \mathbb{C}^{n}\right)$ we can define a function $f_{\mathbf{a}} \in \mathcal{S}^{\prime}\left(\mathbb{R}^{n}\right)$ as follows $f_{\mathbf{a}}(\mathbf{x})=f(\mathbf{x}+\mathbf{a}), \mathbf{x} \in \mathbb{R}^{n}$. Let $P, Q$ be polynomials. Denote by $\top(P, Q, \mathbf{a}) f(\mathbf{x})=$ $P(D) f(\mathbf{x})+Q(D) f_{\mathbf{a}}$ and $\top^{m+1}(P, Q, \mathbf{a}) f(\mathbf{x})=\top(P, Q, \mathbf{a})\left(T^{m}(P, Q, \mathbf{a}) f\right)(\mathbf{x})$ for $m \in \mathbb{Z}_{+}$. Now we have the following result.

Theorem 6. Let $\Phi$ be an arbitrary Young function, $P$ and $Q$ be polynomials and $\mathbf{a} \in \mathbb{R}^{n}$, $f \in L^{\Phi}\left(\mathbb{R}^{n}\right)\left(\right.$ or $\left.\mathbf{a} \in \mathbb{C}^{n}, f \in L^{\Phi}\left(\mathbb{R}^{n}\right) \cap \mathcal{F}\left(\mathcal{E}^{\prime}\left(\mathbb{R}^{n}\right)\right)\right)$. Then $\operatorname{sp}\left(\top^{m}(P, Q, \mathbf{a}) f\right) \subset \operatorname{sp}(f) \forall m \in \mathbb{N}$ and

$$
\liminf _{m \rightarrow \infty}\left\|T^{m}(P, Q, \mathbf{a}) f\right\|_{\Phi}^{1 / m} \geq \sup \left\{\left|P(\mathbf{x})+Q(\mathbf{x}) e^{i \mathbf{a x}}\right|: \mathbf{x} \in \operatorname{sp}(f)\right\}
$$

Proof. It is easy to see $\operatorname{sp}\left(T^{m}(P, Q, \mathbf{a}) f\right) \subset \operatorname{sp}(f) \forall m \in \mathbb{Z}_{+}$from the fact that $\operatorname{sp}\left(D^{\alpha} f\right) \subset$ $\operatorname{sp}(f)$ and $\operatorname{sp}\left(D^{\alpha} f(.+\mathbf{a})\right) \subset \operatorname{sp}(f)$ for all $\alpha \in \mathbb{Z}_{+}^{n}$. Now we prove (18). By the definition of $T^{m}(P, Q, \mathbf{a}) f$, one has $\mathcal{F}\left(T^{m}(P, Q, \mathbf{a}) f\right)=\left(P(\mathbf{x})+Q(\mathbf{x}) e^{i \mathbf{a x}}\right) \mathcal{F}\left(T^{m-1}(P, Q, \mathbf{a}) f\right)$ and then

$$
\mathcal{F}\left(\top^{m}(P, Q, \mathbf{a}) f\right)=\left(P(\mathbf{x})+Q(\mathbf{x}) e^{i \mathbf{a x}}\right)^{m} \widehat{f} \quad \forall m \in \mathbb{N} .
$$


We consider $\varrho \in \operatorname{sp}(f)$ satisfying $\left|P(\varrho)+Q(\varrho) e^{i \mathbf{a} \varrho}\right|>0$. Then, for sufficiently small $\varepsilon>0$, we obtain $\inf \left\{\left|P(\mathbf{x})+Q(\mathbf{x}) e^{i \mathbf{a x}}\right|: \mathbf{x} \in B(\varrho, \varepsilon)\right\}>0$ and there is a function $\psi \in C^{\infty}\left(\mathbb{R}^{n}\right)$, supp $\psi \subset$ $B(\varrho, \varepsilon)$ such that $\langle\widehat{f}, \psi\rangle \neq 0$. We define the function $\mathcal{G}_{m}$ as follows $\mathcal{G}_{m}=\mathcal{F}(\psi(\mathbf{x}) /(P(\mathbf{x})+$ $\left.\left.Q(\mathbf{x}) e^{i \mathbf{a x}}\right)^{m}\right)$. Then $\mathcal{G}_{m}$ is well defined and by (19), we get

$$
\begin{aligned}
& \left\langle\top^{m}(P, Q, \mathbf{a}) f, \mathcal{G}_{m}\right\rangle=\left\langle\top^{m} \widehat{(P, Q, \mathbf{a})} f, \mathcal{F}^{-1}\left(\mathcal{G}_{m}\right)\right\rangle=\left\langle\left(P(\mathbf{x})+Q(\mathbf{x}) e^{i \mathbf{a x}}\right)^{m} \widehat{f}, \mathcal{F}^{-1}\left(\mathcal{G}_{m}\right)\right\rangle \\
& =\left\langle\widehat{f},\left(P(\mathbf{x})+Q(\mathbf{x}) e^{i \mathbf{a x}}\right)^{m} \mathcal{F}^{-1}\left(\mathcal{G}_{m}\right)\right\rangle=\langle\widehat{f}, \psi\rangle
\end{aligned}
$$

and apply Lemma 1 to conclude that

$$
|\langle\widehat{f}, \psi\rangle|=\left|\left\langle T^{m}(P, Q, \mathbf{a}) f, \mathcal{G}_{m}\right\rangle\right| \leq\left\|T^{m}(P, Q, \mathbf{a}) f\right\|_{\Phi}\left\|\mathcal{G}_{m}\right\|_{(\bar{\Phi})} .
$$

Therefore, it follows from $\langle\widehat{f}, \psi\rangle \neq 0$ that

$$
\liminf _{m \rightarrow \infty}\left\|\top^{m}(P, Q, \mathbf{a}) f\right\|_{\Phi}^{1 / m} \geq 1 / \limsup _{m \rightarrow \infty}\left\|\mathcal{G}_{m}\right\|_{(\bar{\Phi})}^{1 / m} .
$$

By the same argument as in the proof of Theorem 2 we get

$$
\limsup _{m \rightarrow \infty}\left\|\mathcal{G}_{m}\right\|_{(\bar{\Phi})}^{1 / m} \leq \sup _{\mathbf{x} \in B(\varrho, \varepsilon)}\left|\left(P(\mathbf{x})+Q(\mathbf{x}) e^{i \mathbf{a x}}\right)^{-1}\right|
$$

and then it follows from (20) that

$$
\liminf _{m \rightarrow \infty}\left\|\top^{m}(P, Q, \mathbf{a}) f\right\|_{\Phi}^{1 / m} \geq 1 / \sup _{\mathbf{x} \in B(\varrho, \varepsilon)}\left|\left(P(\mathbf{x})+Q(\mathbf{x}) e^{i \mathbf{a x}}\right)^{-1}\right| .
$$

Letting $\varepsilon \rightarrow 0$, we obtain $\liminf _{m \rightarrow \infty}\left\|T^{m}(P, Q, \mathbf{a}) f\right\|_{\Phi}^{1 / m} \geq\left|P(\varrho)+Q(\varrho) e^{i \mathbf{a} \varrho}\right|$ and then

$$
\liminf _{m \rightarrow \infty}\left\|\top^{m}(P, Q, \mathbf{a}) f\right\|_{\Phi}^{1 / m} \geq \sup \left\{\left|P(\mathbf{x})+Q(\mathbf{x}) e^{i \mathbf{a x}}\right|: \mathbf{x} \in \operatorname{sp}(f)\right\}
$$

We have the following generalization of (2).

Theorem 7. Let $\Phi$ be an arbitrary Young function, a $\in \mathbb{C}^{n}, P, Q$ be polynomials and $f \in$ $L^{\Phi}\left(\mathbb{R}^{n}\right)$. Assume that $\operatorname{sp}(f)$ is compact. Then $\top^{m}(P, Q, \mathbf{a}) f \in L^{\Phi}\left(\mathbb{R}^{n}\right)$ for all $m$, and

$$
\lim _{m \rightarrow \infty}\left\|T^{m}(P, Q, \mathbf{a}) f\right\|_{\Phi}^{1 / m}=\sup \left\{\left|P(\mathbf{x})+Q(\mathbf{x}) e^{i \mathbf{a x}}\right|: \mathbf{x} \in \operatorname{sp}(f)\right\} .
$$

Proof. Put $K=\operatorname{sp}(f)$. For any $\varepsilon>0$ we choose a function $\mathcal{J} \in C^{\infty}\left(\mathbb{R}^{n}\right)$ satisfying $\mathcal{J}(\mathbf{x})=0$ $\forall \mathbf{x} \notin K_{\varepsilon}$, and $\mathcal{J}(\mathbf{x})=1 \forall \mathbf{x} \in K_{\varepsilon / 2}$. Then it follows from (19) that $\top^{m} \widehat{(P, Q, \mathbf{a}) f}=\mathcal{J}(\mathbf{x})(P(\mathbf{x})+$ $\left.Q(\mathbf{x}) e^{i \mathbf{a x}}\right)^{m} \widehat{f}$ and then

$$
\top^{m}(P, Q, \mathbf{a}) f=(2 \pi)^{-n / 2} f * \mathcal{F}^{-1}\left(\mathcal{J}(\mathbf{x})\left(P(\mathbf{x})+Q(\mathbf{x}) e^{i \mathbf{a x}}\right)^{m}\right) .
$$

Hence, using Lemma 2, we deduce that $\top^{m}(P, Q, \mathbf{a}) f \in L^{\Phi}\left(\mathbb{R}^{n}\right)$ and

$$
\left\|\top^{m}(P, Q, \mathbf{a}) f\right\|_{\Phi} \leq(2 \pi)^{-n / 2}\|f\|_{\Phi}\left\|\mathcal{F}^{-1}\left(\mathcal{J}(\mathbf{x})\left(P(\mathbf{x})+Q(\mathbf{x}) e^{i \mathbf{a x}}\right)^{m}\right)\right\|_{1} .
$$

Therefore,

$$
\limsup _{m \rightarrow \infty}\left\|\top^{m}(P, Q, \mathbf{a}) f\right\|_{\Phi}^{1 / m} \leq \limsup _{m \rightarrow \infty}\left\|\mathcal{F}^{-1}\left(\mathcal{J}(\mathbf{x})\left(P(\mathbf{x})+Q(\mathbf{x}) e^{i \mathbf{a x}}\right)^{m}\right)\right\|_{1}^{1 / m} .
$$


Similarly as in the proof of Theorem 3, we get

$$
\limsup _{m \rightarrow \infty}\left\|\mathcal{F}^{-1}\left(\mathcal{J}(\mathbf{x})\left(P(\mathbf{x})+Q(\mathbf{x}) e^{i \mathbf{a x}}\right)^{m}\right)\right\|_{1}^{1 / m} \leq \sup \left\{\left|P(\mathbf{x})+Q(\mathbf{x}) e^{i \mathbf{a x}}\right|: \mathbf{x} \in K_{\varepsilon}\right\} .
$$

Then it follows from (21) that $\limsup \left\|\top^{m}(P, Q, \mathbf{a}) f\right\|_{\Phi}^{1 / m} \leq \sup \left\{\left|P(\mathbf{x})+Q(\mathbf{x}) e^{i \mathbf{a x}}\right|: \mathbf{x} \in K_{\varepsilon}\right\}$. Letting $\varepsilon \rightarrow 0$ with the note that $K$ is compact, we obtain

$$
\limsup _{m \rightarrow \infty}\left\|\top^{m}(P, Q, \mathbf{a}) f\right\|_{\Phi}^{1 / m} \leq \sup \left\{\left|P(\mathbf{x})+Q(\mathbf{x}) e^{i \mathbf{a x}}\right|: \mathbf{x} \in \operatorname{sp}(f)\right\}
$$

From this and (18), we arrive

$$
\lim _{m \rightarrow \infty}\left\|\top^{m}(P, Q, \mathbf{a}) f\right\|_{\Phi}^{1 / m}=\sup \left\{\left|P(\mathbf{x})+Q(\mathbf{x}) e^{i \mathbf{a x}}\right|: \mathbf{x} \in \operatorname{sp}(f)\right\}
$$

Theorem 8. Let $\Phi$ be an arbitrary Young function, $\mathbf{a} \in \mathbb{C}^{n}, P, Q$ be polynomials, $f \in L^{\Phi}\left(\mathbb{R}^{n}\right)$ and $\Omega:=\left\{\mathbf{x} \in \mathbb{R}^{n}:\left|P(\mathbf{x})+Q(\mathbf{x}) e^{i \mathbf{a x}}\right| \leq 1\right\}$. Assume that $\Omega$ is the compact set. Then $\operatorname{sp}(f) \subset \Omega$ if and only if $\top^{m}(P, Q, \mathbf{a}) f \in L^{\Phi}\left(\mathbb{R}^{n}\right)$ for all $m \in \mathbb{Z}_{+}$and

$$
\liminf _{m \rightarrow \infty}\left\|T^{m}(P, Q, \mathbf{a}) f\right\|_{\Phi}^{1 / m} \leq 1 .
$$

Proof. Necessary. Assume that $\operatorname{sp}(f) \subset \Omega$. Hence, $\operatorname{sp}(f)$ is also compact. Then it follows from Theorem 7 that

$$
\lim _{m \rightarrow \infty}\left\|\top^{m}(P, Q, \mathbf{a}) f\right\|_{\Phi}^{1 / m}=\sup \left\{\left|P(\mathbf{x})+Q(\mathbf{x}) e^{i \mathbf{a x}}\right|: \mathbf{x} \in \operatorname{sp}(f)\right\}
$$

This implies, by $\operatorname{sp}(f) \subset \Omega$, that $\lim _{m \rightarrow \infty}\left\|T^{m}(P, Q, \mathbf{a}) f\right\|_{\Phi}^{1 / m} \leq 1$.

Sufficiency. Assume that (22) holds. Then it follows from Theorem 7 that $\sup \{\mid P(\mathbf{x})+$ $\left.Q(\mathbf{x}) e^{i \mathbf{a x}} \mid: \mathbf{x} \in \operatorname{sp}(f)\right\} \leq 1$ and then $\operatorname{sp}(f) \subset \Omega$.

Theorem 9. Let $\Phi$ be an arbitrary Young function and $K$ be an arbitrary compact set in $\mathbb{R}^{n}$. Then for any $\tau>0$ there exists a constant $C_{\tau, K}<\infty$ independent of $\Phi$ such that

$$
\|\top(P, Q, \mathbf{a}) f\|_{\Phi} \leq C_{\tau, K}\|f\|_{\Phi} \sup _{\mathbf{x} \in K_{(\tau)}}\left|P(\mathbf{x})+Q(\mathbf{x}) e^{i \mathbf{a x}}\right|
$$

for all $\mathbf{a} \in \mathbb{C}^{n}, f \in \mathcal{E}_{\Phi}(K)$ and for all polynomials $P(\mathbf{x}), Q(\mathbf{x})$, where $\mathcal{E}_{\Phi}(K)=\left\{f \in L^{\Phi}\left(\mathbb{R}^{n}\right)\right.$ : $\operatorname{sp}(f) \subset K\}$.

Proof. Necessity. We choose a function $\mathcal{A} \in C_{0}^{\infty}\left(\mathbb{R}^{n}\right)$ such that $\mathcal{A}(\mathbf{z})=1$ if $\mathbf{z} \in K_{\tau / 4}$ and $\mathcal{A}(\mathbf{z})=0$ if $\mathbf{z} \notin K_{\tau / 2}$. It follows from $\mathcal{F}(\top(P, Q, \mathbf{a}) f)=\left(P(\mathbf{z})+Q(\mathbf{z}) e^{i \mathbf{a z}}\right) \widehat{f}$ and $\operatorname{sp}(f) \subset K$ that $\mathcal{F}(T(P, Q, \mathbf{a}) f)=\mathcal{A}(\mathbf{z})\left(P(\mathbf{z})+Q(\mathbf{z}) e^{i \mathbf{a z}}\right) \widehat{f}$, and then

$$
\top(P, Q, \mathbf{a}) f=(2 \pi)^{-n / 2} f * \mathcal{F}^{-1}\left(\mathcal{A}(\mathbf{z})\left(P(\mathbf{z})+Q(\mathbf{z}) e^{i \mathbf{a z}}\right)\right) .
$$

Therefore, by Lemma 2 we have

$$
\|\top(P, Q, \mathbf{a}) f\|_{\Phi} \leq(2 \pi)^{-n / 2}\|f\|_{\Phi}\left\|\mathcal{F}^{-1}\left(\mathcal{A}(\mathbf{z})\left(P(\mathbf{z})+Q(\mathbf{z}) e^{i \mathbf{a z}}\right)\right)\right\|_{1}=(2 \pi)^{-n / 2}\|f\|_{\Phi}\|\mathcal{J}\|_{1},
$$


where $\mathcal{J}(\mathbf{x}):=\left(\mathcal{F}^{-1}\left(\mathcal{A}(\mathbf{z})\left(\left(P(\mathbf{z})+Q(\mathbf{z}) e^{i \mathbf{a z}}\right)\right)\right)(\mathbf{x})\right.$. Hence, for $\sigma \in \mathbb{Z}_{+}^{n}, \sigma \leq(2,2, \ldots, 2)$ we get the following estimate

$$
\begin{aligned}
\sup _{\mathbf{x} \in \mathbb{R}^{n}}\left|\mathbf{x}^{\sigma} \mathcal{J}(\mathbf{x})\right| & =(2 \pi)^{-n / 2} \sup _{\mathbf{x} \in \mathbb{R}^{n}}\left|\int_{\mathbb{R}^{n}} e^{i x \mathbf{z}} D^{\sigma}\left(\mathcal{A}(\mathbf{z})\left(P(\mathbf{z})+Q(\mathbf{z}) e^{i \mathbf{a z}}\right)\right) d \mathbf{z}\right| \\
& =(2 \pi)^{-n / 2} \sup _{\mathbf{x} \in \mathbb{R}^{n}}\left|\int_{K_{\tau / 2}} e^{i x \mathbf{z}} D^{\sigma}\left(\mathcal{A}(\mathbf{z})\left(P(\mathbf{z})+Q(\mathbf{z}) e^{i \mathbf{a z}}\right)\right) d \mathbf{z}\right| \\
& \leq(2 \pi)^{-n / 2} \int_{K_{\tau / 2}}\left|D^{\sigma}\left(\mathcal{A}(\mathbf{z})\left(P(\mathbf{z})+Q(\mathbf{z}) e^{i \mathbf{a z}}\right)\right)\right| d \mathbf{z} .
\end{aligned}
$$

Then it follows from Leibniz's rule that

$$
\begin{aligned}
\sup _{\mathbf{x} \in \mathbb{R}^{n}}\left|\mathbf{x}^{\sigma} \mathcal{J}(\mathbf{x})\right| & \leq(2 \pi)^{-n / 2} \int_{K_{\tau / 2}}\left|\sum_{\gamma \leq \sigma} \frac{\sigma !}{\gamma !(\sigma-\gamma) !} D^{\gamma} \mathcal{A}(\mathbf{z}) D^{\sigma-\gamma}\left(P(\mathbf{z})+Q(\mathbf{z}) e^{i \mathbf{a z}}\right)\right| d \mathbf{z} \\
& \leq(2 \pi)^{-n / 2} \sum_{\gamma \leq \sigma}\left(\frac{\sigma !}{\gamma !(\sigma-\gamma) !} \sup _{\mathbf{x} \in K_{\tau / 2}}\left|D^{\sigma-\gamma}\left(P(\mathbf{x})+Q(\mathbf{x}) e^{i \mathbf{a x}}\right)\right| \int_{K_{\tau / 2}}\left|D^{\gamma} \mathcal{A}(\mathbf{z})\right| d \mathbf{z}\right) \\
& \leq(2 \pi)^{-n / 2} \max _{\nu \leq(2,2, \ldots, 2)} \sup _{\mathbf{x} \in K_{\tau / 2}}\left|D^{\nu}\left(P(\mathbf{x})+Q(\mathbf{x}) e^{i \mathbf{a x}}\right)\right| \\
& \times \sum_{\gamma \leq \sigma}\left(\frac{\sigma !}{\gamma !(\sigma-\gamma) !} \int_{K_{\tau / 2}}\left|D^{\gamma} \mathcal{A}(\mathbf{z})\right| d \mathbf{z}\right) .
\end{aligned}
$$

Because the derivatives of the analytic function $\left(P(\mathbf{x})+Q(\mathbf{x}) e^{i \mathbf{a x}}\right)$ can be estimated in $K_{\tau / 2}$ by the maximum of the modulus in $K_{(\tau)}$, there exists a constant $A_{\tau}<\infty$ independent of $f, P(\mathbf{x})$, $Q(\mathbf{x})$, a, and $\Phi$ such that

$$
\sup _{\mathbf{x} \in K_{\tau / 2}}\left|D^{v}\left(P(\mathbf{x})+Q(\mathbf{x}) e^{i \mathbf{a x}}\right)\right| \leq A_{\tau} \sup _{\mathbf{x} \in K_{(\tau)}}\left|\left(P(\mathbf{x})+Q(\mathbf{x}) e^{i \mathbf{a x}}\right)\right|
$$

for all $v \in \mathbb{Z}_{+}^{n}, v \leq(2,2, \ldots, 2)$. From (25) and (26), we have

$$
\begin{aligned}
\sup _{\mathbf{x} \in \mathbb{R}^{n}}\left|\mathbf{x}^{\sigma} \mathcal{J}(\mathbf{x})\right| & \leq(2 \pi)^{-n / 2} \sum_{\gamma \leq \sigma}\left(\frac{\sigma !}{\gamma !(\sigma-\gamma) !} A_{\tau} \sup _{\mathbf{x} \in K_{(\tau)}}\left|\left(P(\mathbf{x})+Q(\mathbf{x}) e^{i \mathbf{a x}}\right)\right| \int_{K_{\tau / 2}}\left|D^{\gamma} \mathcal{A}(\mathbf{z})\right| d \mathbf{z}\right) \\
& \leq(2 \pi)^{-n / 2} 2^{2 n} A_{\tau} C \sup _{\mathbf{x} \in K_{(\tau)}}\left|P(\mathbf{x})+Q(\mathbf{x}) e^{i \mathbf{a x}}\right|
\end{aligned}
$$

where

$$
C:=\max _{\gamma \leq(2,2, \ldots, 2)} \int_{K_{\tau / 2}}\left|D^{\gamma} \mathcal{A}(\mathbf{z})\right| d \mathbf{z} \text {. }
$$

Then it follows from (27) and $\|\mathcal{J}\|_{1} \leq \pi^{n} \sup _{\mathbf{x} \in \mathbb{R}^{n}}\left|\left(1+x_{1}^{2}\right)\left(1+x_{2}^{2}\right) \ldots\left(1+x_{n}^{2}\right) \mathcal{J}(\mathbf{x})\right|$ that

$$
\|\mathcal{J}\|_{1} \leq C_{\tau, K} \sup _{\mathbf{x} \in K_{(\tau)}}\left|P(\mathbf{x})+Q(\mathbf{x}) e^{i \mathbf{a x}}\right|
$$

where $C_{\tau, K}$ is independent of $f, P(\mathbf{x}), Q(\mathbf{x}), \mathbf{a}, \Phi$. Combining (24) and (28), we obtain (23).

Acknowledgments. The authors would like to thank the referee very much for the valuable remarks. This research is funded by Vietnam National Foundation for Science and Technology Development (NAFOSTED) under grant number 101.02-2018.300. 


\section{References}

[1] Abreu L.D. Real Paley-Wiener theorems for the Koornwinder-Swarttouw q-Hankel transform. J. Math. Anal. Appl. 2007, 334 (1), 223-231. doi:10.1016/j.jmaa.2006.12.050

[2] Albrecht E., Ricker W.J. Local spectral properties of certain matrix differential operators in $L^{p}\left(\mathbb{R}^{N}\right)^{m}$. J. Operator Theory 1996, 35 (1), 3-37.

[3] Andersen N.B. Real Paley-Wiener theorems. Bull. Lond. Math. Soc. 2004, 36 (4), 504-508. doi:10.1112/S0024609304003108

[4] Bang H.H. A property of infinitely differentiable functions. Proc. Amer. Math. Soc. 1990, 108 (1), $73-76$. doi:10.2307/2047695

[5] Bang H.H. Functions with bounded spectrum. Trans. Amer. Math. Soc. 1995, 347 (3), 1067-1080. doi: $10.2307 / 2154890$

[6] Bang H.H. Spectrum of functions in Orlicz spaces. J. Math. Sci. Univ. Tokyo 1997, 4 (2), 341-349.

[7] Bang H.H. The study of the properties of functions belonging to an Orlicz space depending on the geometry of their spectra. Izv. RAN. Ser. Mat. 1997, 61 (2), 163-198. doi:10.4213/im120 (in Russian)

[8] Bang H.H., Huy V.N. Behavior of the sequence of norms of primitives of a function. J. Approx. Theory 2010, 162 (6), 1178-1186. doi:10.1016/j.jat.2009.12.011

[9] Bang H.H., Huy V.N. Behavior of sequences of norms of primitives of functions depending on their spectrum. Dokl. Math. 2011, 84 (2), 672-674. doi:10.1134/S1064562411060263 (translation of Dokl. Akad. Nauk 2011, 440 (4), 456-458. (in Russian))

[10] Bang H.H., Huy V.N. A study of behavior of the sequence of norm of primitives of functions in Orlicz spaces depending on their spectral. Tokyo J. Math. 2015, 38 (1), 283-308. doi:10.3836/tjm/1437506250

[11] Bernstein S.N. Collected works, Vol. II. Izdat. Akad. Nauk SSSR, Moscow, 1954. (in Russian)

[12] Betancor J.J., Betancor J.D., Méndez J.M.R. Paley-Wiener type theorems for Chébli-Trimèche transforms. Publ. Math. Debrecen 2002, 60 (3-4), 347-358.

[13] Chettaoui C., Trimèche K. New type Paley-Wiener theorems for the Dunkl transform on $\mathbb{R}$. Integral Transforms Spec. Funct. 2003, 14 (2), 97-115. doi:10.1080/10652460290029635

[14] De Jeu M. Some remarks on a proof of geometrical Paley-Wiener theorems for the Dunkl transform. Integral Transforms Spec. Funct. 2007, 18 (5), 383-385. doi:10.1080/10652460701320752

[15] De Jeu M. Paley-Wiener theorems for the Dunkl transform. Trans. Amer. Math. Soc. 2006, 358 (10), $4225-4250$.

[16] Dzhaparidze K., Zanten J.H. On Bernstein-type inequalities for martingales. Stochastic Process. Appl. 2001,93 (1), 109-117. doi:10.1016/S0304-4149(00)00086-7

[17] Frappier C., Rahman Q.I. On an inequality of S. Bernstein. Canad. J. Math. 1982, 34 (4), 932-944. doi: 10.4153/CJM-1982-066-7

[18] Hang H., Steinwart I. A Bernstein-type inequality for some mixing processes and dynamical systems with an application to learning. Ann. Statist. 2017, 45 (2), 708-743. doi:10.1214/16-AOS1465

[19] Krasnoselskii M.A., Rutickii Y.B. Convex functions and Orlicz spaces. Gos. Izdat. Fiz.-Mat. Lit., Moscow, 1958. (in Russian)

[20] Luxemburg W. Banach function spaces. Ph.D. Thesis, Technische Hogeschool te Delft., Netherlands, 1955.

[21] Máté A., Nevai P.G. Bernstein's inequality in $L^{p}$ for $0<p<1$ and $(C, 1)$ bounds for orthogonal polynomials. Ann. of Math. 1980, 111 (1), 145-154. doi:10.2307/1971219

[22] Musielak J. Orlicz spaces and modular spaces. In: Dold A., Eckmann B. (Eds.) Lecture Notes in Mathematics, 1034. Springer-Verlag, Berlin, 1983.

[23] Nikolskii S.M. Approximation of functions of several variables and imbedding theorems. Nauka, Moscow, 1977. (in Russian) 
[24] O’Neil R. Fractional integration in Orlicz space. I. Trans. Amer. Math. Soc. 1965, 115, 300-328. doi:10.2307/1994271

[25] Pesenson I. Bernstein-Nikolskii and Plancherel-Polya inequalities in $L^{p}$-norms on noncompact symmetric spaces. Math. Nachr. 2009, 282 (2), 253-269. doi:10.1002/mana.200510736

[26] Rahman Q.I., Tariq Q.M. On Bernstein's inequality for entire functions of exponential type. J. Math. Anal. Appl. 2009, 359 (1), 168-180. doi:10.1016/j.jmaa.2009.05.035

[27] Rahman Q.I., Schmeisser G. $L^{p}$ inequalities for entire functions of exponential type. Trans. Amer. Math. Soc. 1990, 320 (1), 91-103. doi:10.2307/2001753

[28] Rao M.M., Ren Z.D. Theory of Orlicz spaces. M. Dekker, New York, 1991.

[29] Rao M.M., Ren Z.D. Applications of Orlicz spaces. M. Dekker, New York, 2002.

[30] Tuan V.K., Zayed A.I. Paley-Wiener-type theorems for a class of integral transforms. J. Math. Anal. Appl. 2002, 266 (1), 200-226. doi:10.1006/jmaa.2001.7740

[31] Tuan V.K. Spectrum of signals. J. Fourier Anal. Appl. 2001, 7 (3), 319-323. doi:10.1007/BF02511817

[32] Vladimirov V.S. Methods of the theory of generalized functions. Taylor \& Francis, London, 2002.

[33] Yurinskii V.V. Exponential inequalities for sums of random vectors. J. Multivariate Anal. 1976, 6 (4), $473-499$. doi:10.1016/0047-259X(76)90001-4

Банг Г.Г., Гуй В.Н. Аеякі спектральні формули для функиій, породжених диференціальними та інтегральними операторами в просторах Орліиа // Карпатські матем. публ. - 2021. - Т.13, №2. - C. 326-339.

У цій статті ми досліджуємо поведінку послідовності $L^{\Phi}$-норм функцій, які породжені диференціальними та інтегральними операторами за допомогою їхнього спектра (носій перетворення Фур'є функції $f$ називають ії спектром і позначають $\operatorname{sp}(f)$ ). $\Delta$ ля деякого полінома $Q$ ми вводимо поняття $Q$-примітивів, яке стає поняттям примітивів, якщо $Q(x)=x$, і вивчаємо поведінку послідовності норм $Q$-примітивів функцій у просторі Орліча $L^{\Phi}\left(\mathbb{R}^{n}\right)$. Ми отримали наступний головний результат: нехай $\Phi$ - довільна функція Юнга, $Q(\mathbf{x})$ - поліном та $\left(\mathcal{Q}^{m} f\right)_{m=0}^{\infty} \subset L^{\Phi}\left(\mathbb{R}^{n}\right)$ задовольняє $\mathcal{Q}^{0} f=f, Q(D) \mathcal{Q}^{m+1} f=\mathcal{Q}^{m} f$ для $m \in \mathbb{Z}_{+}$. Припустимо, шо $\operatorname{sp}(f)$ є компактом і $\operatorname{sp}\left(\mathcal{Q}^{m} f\right)=\operatorname{sp}(f)$ для всіх $m \in \mathbb{Z}_{+}$. Тоді

$$
\lim _{m \rightarrow \infty}\left\|\mathcal{Q}^{m} f\right\|_{\Phi}^{1 / m}=\sup _{\mathbf{x} \in \operatorname{sp}(f)}|1 / Q(\mathbf{x})| .
$$

Подано також віАповідні результати для функцій, що породжені диференціальними та інтегральними операторами.

Ключові слова і фрази: простір Орліча, нерівність в апроксимації, перетворення Фур'є, узагальнена функція. 